\title{
SPECTRAL EFFICIENCY OF CDMA UPLINK CELLULAR NETWORKS
}

\author{
Nicolas Bonneau ${ }^{1}$, Mérouane Debbah ${ }^{2}$, Eitan Altman ${ }^{1}$ and Giuseppe Caire ${ }^{2}$ \\ ${ }^{1}$ MAESTRO, INRIA Sophia Antipolis, 2004 Route des Lucioles, B.P.93 06902 Sophia Antipolis, France \\ ${ }^{2}$ Mobile Communications Group, Institut Eurecom, 2229 Route des Cretes, B.P.193 06904 Sophia Antipolis, France \\ E-mail: \{nbonneau, altman\}@sophia.inria.fr, \{debbah, caire\}@eurecom. fr
}

\begin{abstract}
In this contribution, the performance of an uplink CDMA system with random spreading and multi-cell interference is analyzed. A useful framework is provided in order to determine the base station coverage for wireless flat fading channels with very dense networks (in the number of users per meter) considering different receiver structures at the base station, namely the Matched filter, the Wiener filter and the Optimum filter. Using asymptotic arguments , analytical expressions of the spectral efficiency are obtained and provide a simple expression of the network capacity based only on a few meaningful parameters.
\end{abstract}

\section{INTRODUCTION}

In the study of CDMA networks, an important problem concerns the optimal deployment of base stations to cope with the amount of traffic. Assuming that a base station has only access to the users within its cell, the gain provided by reducing the cell size is studied. Previous works have already studied the capacity of an uplink CDMA multi-cell network with various interference models [1, 2, 3]. For example, [1] takes into account only the interference of two adjacent cells in a linear setting, according to Wyner's model, while [2] restricts the interference to a portion of the plane. However, none has taken explicitly into account the cumulative effect from all interfering cells with realistic path loss models. This contribution analyzes this setting for three types of receiver structures: Matched filter, Wiener filter and Optimum filter. In particular, a new approach, already proposed in the downlink case in [4], is used to model the network given a uniformly distributed density $d$ (number of users per meter) of the users as well as certain characteristics of the fading channel. In order to obtain interpretable expressions, the problem is analyzed in the asymptotic regime: very dense networks are considered where the spreading length $N$ tends to infinity, $d$ tends to infinity but the ratio $\frac{d}{N} \rightarrow \alpha$ is constant. The effect of shadowing albeit important, is neglected. The results are mainly based on random matrix theory $[5,6]$. One of the great features of this tool is that performance measures such as SINR or spectral efficiency have very simple forms in the large system limit, independent of the particular CDMA code structure. Moreover, the theoretical results were shown to be very accurate predictions of the system's behavior in the finite size case (spreading length $N$ of 256).

This paper is structured as follows: In section 2, the CDMA cellular model is introduced. In section 3, the SINR (Signal to Interference plus Noise ratio) expression is derived and an asymptotic analysis of the spectral efficiency with Matched filter, Wiener filter and optimum filter is provided. Finally in section 4 , discussions as well as numerical simulations are provided in order to validate our analysis.

\section{UPLINK CDMA CELLULAR MODEL}

\subsection{Cellular Model}

We focus our analysis on a one dimensional (1D) network. This scenario represents for example the case of the deployment of base stations along a motorway (users i.e cars move along the motorway). An infinite length $((2 L+1) a$ with $L \rightarrow \infty$ ) base station deployment is considered (see figure 1). The base stations are supposed equidistant with interbase station distance $a$. The spreading length $N$ is fixed and is independent of the number of users. The number of users per cell and the load of each cell are respectively given by $K=d * a$ and $\alpha a$.

\subsection{Uplink CDMA Model}

In the following, upper and lower boldface symbols will be used for matrices and column vectors, respectively. (.) ${ }^{H}$ will denote hermitian transpose. The abbreviation a.s. means almost surely. The $N \times 1$ received signal $\mathbf{y}$ at the base station has the form:

$$
\mathbf{y}=\mathbf{W P s}+\mathbf{W}_{+} \mathbf{P}_{+} \mathbf{s}_{+}+\mathbf{n}
$$

WPs is the useful signal, transmitted by users within the cell whereas $\mathbf{W}_{+} \mathbf{P}_{+} \mathbf{s}_{+}$represents the inter-cell interference. $\mathbf{W}$ and $\mathbf{W}_{+}$are respectively $N \times K$ and $N \times 2 L K$ 


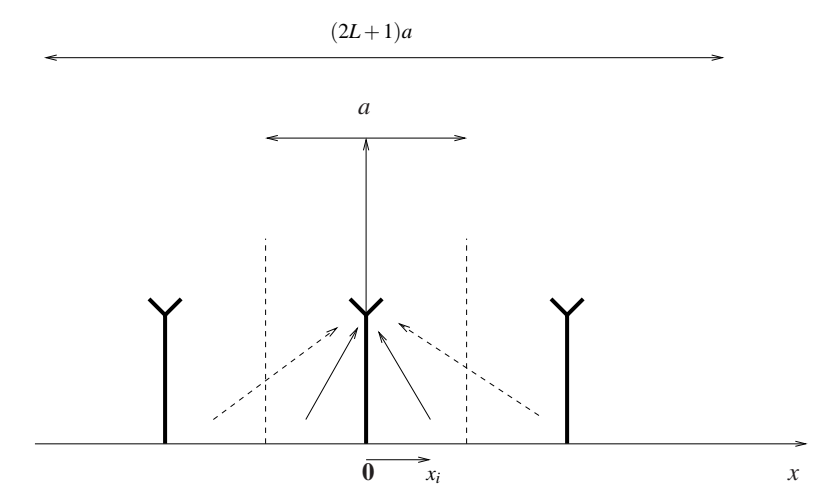

Fig. 1. Representation of a large scale network.

random spreading matrices, with i.i.d. entries (for example in $\left\{\frac{-1}{\sqrt{N}}, \frac{1}{\sqrt{N}}\right\}$ ). $\mathbf{n}$ is an $N \times 1$ Additive White Gaussian Noise (AWGN) vector with variance $\sigma^{2}$. Any user $i$ is determined by his position $x_{i} . \mathbf{P}$ and $\mathbf{P}_{+}$are the power attenuation diagonal matrices, respectively of size $K \times K$ and $2 L K \times 2 L K$. Their general form is:

$$
\begin{gathered}
\mathbf{P}=\operatorname{diag}\left[h_{1} \sqrt{P\left(x_{1}\right)}, \ldots, h_{K} \sqrt{P\left(x_{K}\right)}\right] \\
\mathbf{P}_{+}=\operatorname{diag}\left[h_{K+1} \sqrt{P\left(x_{K+1}\right)}, \ldots, h_{(2 L+1) K} \sqrt{P\left(x_{(2 L+1) K}\right)}\right]
\end{gathered}
$$

where $\left\{h_{k}\right\}_{k=1 \ldots(2 L+1) K}$ and $P(x)$ represent respectively flat fading and path loss.

\subsection{Performance metric}

We would like to quantify the number of bits/s/Hz the system is able to deliver to all the users given a certain intercell distance. The users are assumed to employ Gaussian codebooks. Due to invariance by translation, the spectral efficiency per cell is the same for all cells. Since the network capacity is infinite, the measure of performance in this case is the number of bits per second per hertz per meter (bits/s/Hz/m) the system is able to deliver defined by:

$$
\gamma=\frac{1}{N T a} I(\mathbf{s}, \mathbf{y})
$$

where $T$ is the chip time (set to 1 in the rest of the paper) and $I(\mathbf{s}, \mathbf{y})$ is the mutual information between the received signal and the transmitted signal for a given receiver structure. In this case, the network capacity is a linear scaling factor of $\gamma$.

\section{SPECTRAL EFFICIENCY}

\subsection{Matched Filter}

Without loss of generality, let us focus on user 1 and denote:

$$
\mathbf{W}=\left[\mathbf{w}_{1} \mid \mathbf{W}^{\complement}\right], \mathbf{P}=\left[\begin{array}{ll}
h_{1} \sqrt{P\left(x_{1}\right)} & \\
& \mathbf{P}^{\complement}
\end{array}\right], \mathbf{s}=\left[\begin{array}{l}
s^{1} \\
\mathbf{s}^{\complement}
\end{array}\right]
$$

where $\mathbf{w}_{1}$ and $s^{1}$ correspond respectively to the first column of $\mathbf{W}$ and the signal of user 1 . The received signal at the output of the matched filter is given by:

$$
\begin{gathered}
\mathbf{w}_{1}^{H} \mathbf{y}=h_{1} \sqrt{P\left(x_{1}\right)} s^{1}+\mathbf{w}_{1}^{H} \mathbf{W}_{+}^{\complement} \mathbf{P}_{+}^{\complement}\left[\begin{array}{c}
\mathbf{s}^{\complement} \\
\mathbf{s}_{+}
\end{array}\right]+\mathbf{w}_{1}^{H} \mathbf{n} \\
\operatorname{SINR}\left(x_{1}\right)=\frac{\left|h_{1}\right|^{2} P\left(x_{1}\right)}{\sigma^{2}+\mathbf{w}_{1}^{H} \mathbf{W}_{+}^{\complement} \mathbf{P}_{+}^{\complement} \mathbf{P}_{+}^{\complement}{ }^{H} \mathbf{W}_{+}^{\complement}{ }^{H} \mathbf{w}_{1}}
\end{gathered}
$$

where $\mathbf{W}_{+}^{\complement}=\left[\mathbf{W}^{\complement} \mid \mathbf{W}_{+}\right]$and $\mathbf{P}_{+}^{\complement}=\left[\begin{array}{ll}\mathbf{P}^{\complement} & \\ & \mathbf{P}_{+}\end{array}\right]$. The spectral efficiency is given by:

$$
\gamma=\frac{1}{N a} \sum_{i=1}^{K} \log _{2}\left(1+\operatorname{SINR}\left(x_{i}\right)\right)
$$

Proposition 1 When $N \rightarrow \infty$ and $\frac{d}{N} \rightarrow \alpha$, the mean spectral efficiency with i.i.d random spreading and Matched filter is:

$$
\begin{aligned}
\mathbb{E}_{h, P(x)}[\gamma] & =\frac{2 \alpha}{a} \int_{0}^{+\infty} \int_{0}^{a / 2} \log _{2}\left(1+\frac{t P(x)}{\sigma^{2}+I}\right) p(t) d x d t \\
\text { where } p(t) & =\mathbb{P}\left(|h|^{2}=t\right) \text { and } \\
I & =2 \alpha \int_{0}^{+\infty} \int_{0}^{+\infty} t^{\prime} p\left(t^{\prime}\right) P\left(x^{\prime}\right) d x^{\prime} d t^{\prime}
\end{aligned}
$$

It is quite straightforward to see that for any decreasing path loss function $P(x)$, the optimum inter-cell distance is $a=0$.

Proof Let $\mathbf{R}_{+}^{\complement}=\mathbf{W}_{+}^{\complement} \mathbf{P}_{+}^{\complement} \mathbf{P}_{+}^{\complement}{ }^{H} \mathbf{W}_{+}^{\complement}{ }^{H}$. It can be shown (see [6]) that:

$$
\begin{aligned}
& \text { 1. } \mathbf{w}_{1}^{H} \mathbf{R}_{+}^{\complement} \mathbf{w}_{1} \underset{N \rightarrow \infty}{\longrightarrow} \frac{1}{N} \operatorname{Trace}\left(\mathbf{R}_{+}^{\complement}\right) \text { a.s. } \\
& \text { 2. } \frac{1}{N} \operatorname{Trace}\left(\mathbf{R}_{+}^{\complement}\right)=\frac{1}{N} \sum_{j=2}^{(2 L+1) K}\left|h_{j}\right|^{2} P\left(x_{j}\right)
\end{aligned}
$$

The spectral efficiency is therefore given by:

$$
\gamma=\frac{1}{N a} \sum_{i=1}^{K} \log _{2}\left(1+\frac{\left|h_{i}\right|^{2} P\left(x_{i}\right)}{\sigma^{2}+\left.\frac{1}{N} \sum_{\substack{(2 L+1) K \\ j \neq i}}^{(2 L+1} h_{j}\right|^{2} P\left(x_{j}\right)}\right)
$$

\subsection{MMSE receiver}

Let $\mathbf{R}^{\complement}=\mathbf{W}^{\complement} \mathbf{P}^{\complement} \mathbf{P}^{\complement}{ }^{H} \mathbf{W}^{\complement}{ }^{H}, \mathbf{R}=\mathbf{W} \mathbf{P} \mathbf{P}^{H} \mathbf{W}^{H}, \mathbf{R}_{+}=$ $\mathbf{W}_{+} \mathbf{P}_{+} \mathbf{P}_{+}^{H} \mathbf{W}_{+}^{H}, \mathbf{F}=\mathbf{R}^{\complement}+\sigma^{2} \mathbf{I}$ and $\mathbf{R}^{\text {tot }}=\mathbf{R}+\mathbf{R}_{+}$.

\subsubsection{Full knowledge of the covariance interference ma-} trix

The output SINR of the Wiener conditioned on the noise variance and the covariance matrix of the intra-cell interference and inter-cell interference is given by (see [7] ${ }^{1}$ :

$$
\operatorname{SINR}\left(x_{1}\right)=\left|h_{1}\right|^{2} P\left(x_{1}\right) \mathbf{w}_{1}^{H}\left(\mathbf{F}+\mathbf{R}_{+}\right)^{-1} \mathbf{w}_{1}
$$

\footnotetext{
${ }^{1}$ The base station is supposed to have a statistical estimate of the interference covariance matrix.
} 
Proposition 2 When $N \rightarrow \infty$ and $\frac{d}{N} \rightarrow \alpha$, the mean spectral efficiency with random spreading and Wiener filter is:

$$
\begin{gathered}
\mathbb{E}_{h, P(x)}[\gamma]= \\
\frac{2 \alpha}{a} \int_{0}^{+\infty} \int_{0}^{a / 2} \log _{2}\left(1+t P(x) m^{\mathbf{R}^{\mathrm{tot}}}\left(-\sigma^{2}\right)\right) p(t) d x d t
\end{gathered}
$$

where $m^{\mathbf{R}^{\text {tot }}}(z)$ is the Stieltjes transform ${ }^{2}$ of the empirical distribution function of the eigenvalues of $\mathbf{R}^{\text {tot }}$ given by [6]:

$$
m^{\mathbf{R}^{\mathrm{tot}}}(z)=\frac{1}{2 \alpha \int_{0}^{+\infty} \int_{0}^{+\infty} \frac{t p(t) P(x) d x d t}{1+t P(x) m^{\mathbf{R}^{\mathrm{tot}}}(z)}-z}
$$

Proof The prooffollows a two step procedure:

$$
\begin{aligned}
& \text { 1. } \mathbf{w}_{1}^{H}\left(\mathbf{F}+\mathbf{R}_{+}\right)^{-1} \mathbf{w}_{1} \underset{N \rightarrow \infty}{\longrightarrow} \frac{1}{N} \operatorname{Trace}\left(\left(\mathbf{F}+\mathbf{R}_{+}\right)^{-1}\right) \text { a.s. } \\
& \text { 2. } \frac{1}{N} \operatorname{Trace}\left(\left(\mathbf{F}+\mathbf{R}_{+}\right)^{-1}\right) \rightarrow \frac{1}{N} \sum_{i=1}^{N} \frac{1}{\lambda_{i}^{\mathbf{R}^{\mathrm{tot}}}+\sigma^{2}} \\
& \frac{1}{N} \sum_{i=1}^{N} \frac{1}{\lambda_{i}^{\mathbf{R}^{\mathrm{tot}}}+\sigma^{2}}=\int \frac{1}{\lambda+\sigma^{2}} \frac{1}{N} \sum_{i=1}^{N} \delta\left(\lambda-\lambda_{i}^{\mathbf{R}^{\mathrm{tot}}}\right) d \lambda \\
& \rightarrow \int \frac{1}{\lambda+\sigma^{2}} d F^{\mathbf{R}^{\mathrm{tot}}}(\lambda)=m^{\mathbf{R}^{\mathrm{tot}}}\left(-\sigma^{2}\right)
\end{aligned}
$$

where $\left\{\lambda_{i}^{\mathbf{R}^{\text {tot }}}\right\}_{i=1 \ldots N}$ are the eigenvalues of $\mathbf{R}^{\mathrm{tot}}$, and $F^{\mathbf{R}^{\mathrm{tot}}}$ is the empirical distribution function of these eigenvalues, i.e. $F^{\mathbf{R}^{\text {tot }}}(\lambda)$ is the proportion of eigenvalues that are inferior or equal to $\lambda$.

\subsubsection{Partial knowledge of the covariance interference ma-} trix

The output SINR of the Wiener filter conditioned on the noise variance and the covariance matrix of the intra-cell interference (the base station knows the signature sequences only of the users within the cell) is given by:

$$
\operatorname{SINR}\left(x_{1}\right)=\frac{\left|h_{1}\right|^{2} P\left(x_{1}\right)\left(\mathbf{w}_{1}^{H} \mathbf{F}^{-1} \mathbf{w}_{1}\right)^{2}}{\mathbf{w}_{1}^{H} \mathbf{F}^{-1} \mathbf{w}_{1}+\mathbf{w}_{1}^{H} \mathbf{F}^{-1} \mathbf{R}_{+} \mathbf{F}^{-1} \mathbf{w}_{1}}
$$

Contrarily to the full knowledge Wiener filter (where it is straightforward that $a=0$ is the optimum inter-cell distance), as the cell size increases, the partial Wiener filter reduces inter-cell interference at the expense of reduced path gain. Note also that when $a \rightarrow \infty$, the partial Wiener filter is equivalent to the full knowledge filter.

Proposition 3 When $N \rightarrow \infty$ and $\frac{d}{N} \rightarrow \alpha$, the mean spectral efficiency with i.i.d Gaussian random spreading and Wiener filter is:

$$
\begin{gathered}
\mathbb{E}_{h, P(x)}[\gamma]= \\
\frac{2 \alpha}{a} \int_{0}^{+\infty} \int_{0}^{a / 2} \log _{2}\left(1+\frac{t P(x) m^{\mathbf{R}^{\complement}}\left(-\sigma^{2}\right)^{2}}{m^{\mathbf{R}^{\complement}}\left(-\sigma^{2}\right)+I}\right) p(t) d x d t
\end{gathered}
$$

\footnotetext{
${ }^{2}$ The Stieltjes transform $m(z)$ of a distribution $G$ is defined as: $m(z)=\int \frac{1}{\lambda-z} d G(\lambda)$
}

where $m^{\mathbf{R}^{\mathrm{C}}}(z)$ is the Stieltjes transform of the empirical distribution function of the eigenvalues of $\mathbf{R}^{\complement}$ given by [6]:

$$
\begin{gathered}
m^{\mathbf{R}^{\complement}}(z)=\frac{1}{2 \alpha \int_{0}^{+\infty} \int_{0}^{a / 2} \frac{t p(t) P(x) d x d t}{1+t P(x) m^{\mathbf{C}}(z)}-z} \\
\text { and } I=2 \alpha \int_{0}^{+\infty} \int_{a / 2}^{+\infty} t p(t) P(x) d x d t .\left[-\frac{\partial m^{\mathbf{R}^{\complement}}(z)}{\partial z}\right]_{-\sigma^{2}}
\end{gathered}
$$

Proof As previously, one shows that:

$$
\begin{gathered}
\mathbf{w}_{1}^{H} \mathbf{F}^{-1} \mathbf{w}_{1} \underset{N \rightarrow \infty}{\longrightarrow} \frac{1}{N} \operatorname{Trace}\left(\mathbf{F}^{-1}\right) \rightarrow m^{\mathbf{R}^{\complement}}\left(-\sigma^{2}\right) \\
\mathbf{w}_{1}^{H} \mathbf{F}^{-1} \mathbf{R}_{+} \mathbf{F}^{-1} \mathbf{w}_{1} \underset{N \rightarrow \infty}{\longrightarrow} \frac{1}{N} \operatorname{Trace}\left(\mathbf{F}^{-2} \mathbf{R}_{+}\right) \text {a.s. }
\end{gathered}
$$

The derivation of the last term uses specific tools of Free Probability Theory [5]. In particular, since $\mathbf{R}^{\complement}$ and $\mathbf{R}_{+}$are almost surely asymptotically free, we have:

$\frac{1}{N} \operatorname{Trace}\left(\mathbf{F}^{-2} \mathbf{R}_{+}\right) \underset{N \rightarrow \infty}{\longrightarrow} \frac{1}{N} \operatorname{Trace}\left(\mathbf{F}^{-2}\right) \frac{1}{N} \operatorname{Trace}\left(\mathbf{R}_{+}\right)$a.s. where

$$
\begin{gathered}
\frac{1}{N} \operatorname{Trace}\left(\mathbf{F}^{-2}\right)=\frac{1}{N} \sum_{i=1}^{N} \frac{1}{\left(\lambda^{\left.\mathbf{R}^{\mathbf{C}}+\sigma^{2}\right)^{2}} \rightarrow\left[-\frac{\partial m^{\mathbf{R}^{\mathbf{C}}}(z)}{\partial z}\right]_{-\sigma^{2}}\right.} \\
\frac{1}{N} \operatorname{Trace}\left(\mathbf{R}_{+}\right)=\frac{1}{N} \sum_{j=K+1}^{(2 L+1) K}\left|h_{j}\right|^{2} P\left(x_{j}\right)
\end{gathered}
$$

\subsection{Optimum receiver}

The mutual information between $\mathbf{y}$ and $\mathbf{s}$ at the output of the optimum receiver (based only on the knowledge of the intra-cell signatures) is given by:

$$
\begin{aligned}
I(\mathbf{s}, \mathbf{y}) & =H(\mathbf{y})-H(\mathbf{y} / \mathbf{s}) \\
& =\log _{2} \operatorname{det}\left(\mathbf{R}^{\text {tot }}+\sigma^{2} I\right)-\log _{2} \operatorname{det}\left(\mathbf{R}_{+}+\sigma^{2} I\right)
\end{aligned}
$$

Proposition 4 When $N \rightarrow \infty$ and $\frac{d}{N} \rightarrow \alpha$, the spectral efficiency with i.i.d random spreading and optimum filter is:

$$
\gamma=\frac{1}{a \ln (2)} \int_{+\infty}^{\sigma^{2}}\left(m^{\mathbf{R}^{\text {tot }}}(z)-m^{\mathbf{R}_{+}}(z)\right) d z
$$

where $m^{\mathbf{R}_{+}}(z)$ is the Stieltjes transforms of the empirical distribution functions of the eigenvalues of $\mathbf{R}_{+}$given by:

$$
m^{\mathbf{R}_{+}}(z)=\frac{1}{2 \alpha \int_{0}^{+\infty} \int_{a / 2}^{+\infty} \frac{t p(t) P(x) d x d t}{1+t P(x) m^{\mathbf{R}^{+}(z)}}-z}
$$

Proof Note that

$$
\begin{aligned}
& \gamma=\frac{1}{N a} \sum_{i=1}^{N} \log _{2}\left(\lambda_{i}^{\mathbf{R}^{\mathrm{tot}}}+\sigma^{2}\right)-\log _{2}\left(\lambda_{i}^{\mathbf{R}_{+}}+\sigma^{2}\right) \\
& \underset{N \rightarrow \infty}{\longrightarrow} \frac{1}{a} \int \log _{2}\left(\lambda+\sigma^{2}\right)\left(d F^{\mathbf{R}^{\mathrm{tot}}}(\lambda)-d F^{\mathbf{R}_{+}}(\lambda)\right)
\end{aligned}
$$


where $\left\{\lambda_{i}^{\mathbf{R}^{\text {tot }}}\right\}_{i=1 \ldots N}$ and $\left\{\lambda_{i}^{\mathbf{R}_{+}}\right\}_{i=1 \ldots N}$ are the sets of eigenvalues of $\mathbf{R}^{\text {tot }}$ and $\mathbf{R}_{+}$, and $F^{\mathbf{R}^{\text {tot }}}$ and $F^{\mathbf{R}_{+}}$are the empirical distribution functions of the eigenvalues. If we derive this expression with respect to $\sigma^{2}$ we obtain:

$$
\frac{\partial \gamma}{\partial \sigma^{2}}=\frac{1}{a \ln 2}\left(m^{\mathbf{R}^{\mathrm{tot}}}\left(-\sigma^{2}\right)-m^{\mathbf{R}_{+}}\left(-\sigma^{2}\right)\right)
$$

where $m^{\mathbf{R}^{\text {tot }}}$ (respectively $m^{\mathbf{R}_{+}}$) is the Stieltjes transform of $F^{\mathbf{R}^{\text {tot }}}$ (resp. $F^{\mathbf{R}_{+}}$).

Note that in the case of no inter-cell interference, the asymptotic spectral efficiency is given by:

$$
\gamma=\frac{1}{a \ln (2)} \int_{+\infty}^{\sigma^{2}}\left(m^{\mathbf{R}^{\complement}}(z)-\frac{1}{z}\right) d z
$$

\section{SIMULATIONS}

In the following, we consider two special fading cases.

1. The unfaded case i.e $p(t)=\delta(t-1)(\delta$ is the Dirac function)

2. The Rayleigh fading case i.e $p(t)=\exp (-t)$.

The path loss is of the polynomial type $P(x)=1 /(|x|+$ $1)^{\beta}$. In figure 2 , we have plotted the spectral efficiency of the matched filter, the Wiener filter and the optimum filter for $\beta=2, \alpha=1$ and $\sigma^{2}=10^{-7}$. Contrary to the case for downlink CDMA studied in [4], spectral efficiency always decreases with inter-cell distance. One can see that the fading does not have a great impact as faded and unfaded curves are very close. Additionally, the curves show that optimum intra-cell processing can more than double the spectral efficiency with respect to the use of the matched filter or the Wiener filter. The relative gap is even higher for increasing inter-cell distance (in which case one is in an overloaded cell system). Note also that exploiting the intercell interference statistics can greatly improve the performance of the Wiener filter (close to the optimum intra-cell processing). In fact, the curve given by equation (4) (not plotted due to scaling factors) shows that inter-cell interference reduces spectral efficiency by a factor of 3 for the range of values of $a$ considered in figure 2 .

\section{CONCLUSION}

Using asymptotic arguments, an explicit expression of the spectral efficiency of multi-cell networks has been derived considering realistic path loss and fading models. We have shown in particular the potential gain in cellular environments of optimum intra-cell processing with respect to various receivers. The impact of inter-cell interference has also been quantified for various inter-cell distances. The results are especially useful for the deployment of cellular

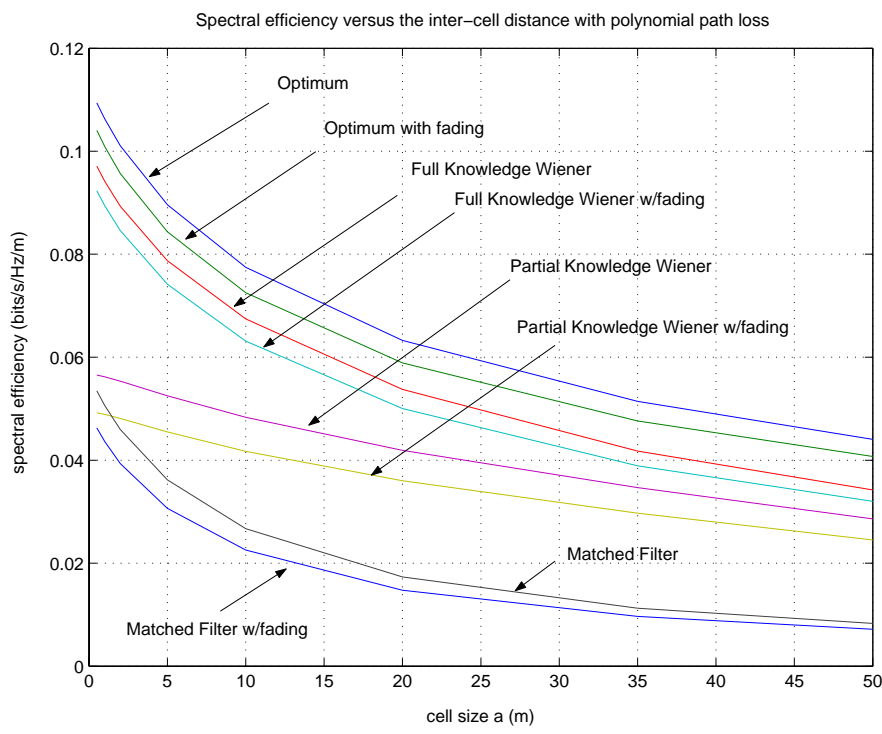

Fig. 2. Results for $\alpha=0.01, P(x)=1 /(|x|+1)^{\beta}, \beta=2$.

networks for a given target user rate. Note that although the model under consideration applies to 1-D networks, it is straightforward to extend the analysis to 2-D networks (in the case of a regular pattern). The effect of frequency selective fading as well as frequency reuse are still being studied.

\section{REFERENCES}

[1] S. Shamai, S.Verdu, and B.M Zaidel, "Multicell uplink spectral efficiency of coded ds-cdma with random signatures," Selected Areas in Communications, IEEE Journal on, vol. 19, pp. 1556-1569, 2001.

[2] S. Hanly and R. Mathar, "On the optimal base-station density for cdma cellular networks," IEEE Trans. on Communications, vol. 50, no. 8, pp. 1274-1281, August 2002.

[3] O. Somekh, B.J. Zaidel, and S. Shamai, "Spectral efficiency of joint multiple cell-site processors for randomly spread dscdma systems," in IEEE International Symposium on Information Theory, Chicago, USA, June 2004.

[4] M. Debbah, "Downlink cdma: to cell or not to cell," in 12th European Signal Processing Conferenc (EUSIPCO 2004), Vienna, Austria, sept. 2004.

[5] D.V. Voiculescu, K.J. Dykema, and A. Nica, Free Random Variables, American Mathematical Society, CRM Monograph Series, Volume 1, Providence, Rhode Island, USA, 1992.

[6] J.W. Silverstein and Z.D. Bai, "On the Empirical Distribution of Eigenvalues of a Class of Large Dimensional Random Matrices," J. Multivariate Anal., vol. 54, no. 2, pp. 175-192, 1995.

[7] D.N.C Tse and S. Hanly, "Linear multi-user receiver: Effective interference, effective bandwidth and user capacity," IEEE Trans. on Information Theory, pp. 641-657, Mar. 1999. 